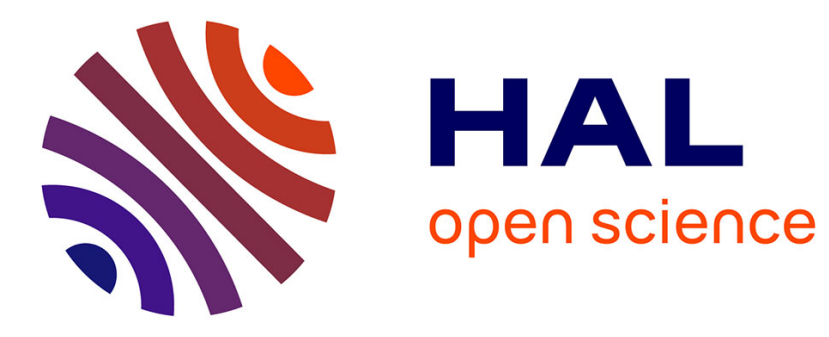

\title{
Pyramidal Algorithms for Littlewood-Paley Decompositions
}

Maria Amelia Muschietti, Bruno Torresani

\section{To cite this version:}

Maria Amelia Muschietti, Bruno Torresani. Pyramidal Algorithms for Littlewood-Paley Decompositions. SIAM Journal on Mathematical Analysis, 1995, 26 (4), pp.925-943. 10.1137/S003614109325222X . hal-01279512

\section{HAL Id: hal-01279512 \\ https://hal.science/hal-01279512}

Submitted on 26 Feb 2016

HAL is a multi-disciplinary open access archive for the deposit and dissemination of scientific research documents, whether they are published or not. The documents may come from teaching and research institutions in France or abroad, or from public or private research centers.
L'archive ouverte pluridisciplinaire HAL, est destinée au dépôt et à la diffusion de documents scientifiques de niveau recherche, publiés ou non, émanant des établissements d'enseignement et de recherche français ou étrangers, des laboratoires publics ou privés. 


\title{
PYRAMIDAL ALGORITHMS FOR LITTLEWOOD-PALEY DECOMPOSITIONS
}

\author{
M.A. MUSCHIETTI ${ }^{\dagger}$ AND B. TORRÉSANI ${ }^{\ddagger}$
}

\begin{abstract}
It is well known that with any usual multiresolution analysis of $L^{2}(I R)$ is associated a pyramidal algorithm for the computation of the corresponding wavelet coefficients. It is shown that an approximate pyramidal algorithm may be associated with more general Littlewood-Paley decompositions. Accuracy estimates are provided for such approximate algorithms. Finally, some explicit examples are studied.
\end{abstract}

1. Introduction. Wavelet analysis has emerged in the past ten years as a completely generic methodology for solving problems in many different areas such as mathematical analysis and operator theory, numerical analysis, signal and image processing, computer vision, computer musics, turbulence, astrophysics for instance. Among the advantages of wavelet decompositions, their relative simplicity and the existence of associated fast algorithms are ones of the most important [1] [6].

There exist essentially two different approaches to wavelets, namely the discrete and the continuous ones. Roughly speaking, the discrete wavelet decompositions are most adapted to problems in which it is important to reduce the volume of data, for instance in signal or image compression, or numerical analysis. On the other hand, for physical signal analysis problems, one is interested in keeping redundancy on the wavelet transform, to get a finer analysis.

The main drawback of continuous wavelet decompositions is that there is a priori no associated fast algorithm for the computation of the corresponding wavelet transform. Some attempts have been made in order to cure such a drawback, mainly by matching a multiresolution framework to the continuous setting (see for instance [4] [7]). They are in general associated with limited classes of wavelets.

We describe here a method for associating fast algorithms to continuous wavelet decompositions, based on the same philosophy. It is in particular shown that starting from an usual mother wavelet, the scale discretization yields a new wavelet (called the integrated wavelet) to which is associated a pair or low and high-pass filters. These filters are in general not discrete, but may in some situations be well approximated by discrete filters, the localization of which can be directly related to the regularity of the scaling function.

The paper is organized as follows. Section 2 is devoted to a description the version of continuous wavelet decompositions that we will use in the sequel. In section 3 we present the algorithmic aspects we are interested in, and in particular our main result theorem (3.2). We present some examples in section 4 and section 5 is devoted to conclusion.

Throughout this paper we shall use the following notations. We shall denote by $\|\cdot\|_{p}$ the $L^{p}(\mathbb{R}), L^{p}([-\pi, \pi])$ and the $\ell^{p}(\mathbb{Z})$ norms. The $L^{p}([-\pi, \pi])$ norm is normalized as:

$$
\|f\|_{p}=\left(\int_{[-\pi, \pi]}|f(x)|^{p} d x\right)^{\frac{1}{p}}
$$

\footnotetext{
† Departamento de Matemática, UNLP, Argentina

¥ GDR "Ondelettes", CNRS
} 
Our conventions for Hermitian product and Fourier transform in $L^{2}(I R)$ are the following ones:

$$
\langle f, g\rangle=\int f(x) g(x)^{*} d x
$$

where the star denotes complex conjugation, and

$$
\hat{f}(\xi)=\int_{\mathbb{R}} f(x) e^{-i \xi x} d x
$$

2. Continuous wavelet decompositions. Let us start from standard notions of continuous wavelet analysis. We will focus on the analysis of $L^{2}(I R)$, and sometimes describe in a few words the corresponding results in the $H^{2}(I R)$ context (we will denote here by $H^{2}(I R)=\left\{f \in L^{2}(I R), \hat{f}(\xi)=0 \forall \xi \leq 0\right\}$ the complex Hardy space). We shall be interested here in two "decomposition-reconstruction" schemes, corresponding to different "reconstruction wavelets".

2.1. The bilinear scheme. Generically, an infinitesimal wavelet (or mother wavelet) is a function $\psi \in L^{1}(I R)$ such that the following admissibility condition holds:

$$
c_{\psi}=\int_{0}^{\infty}|\hat{\psi}(u)|^{2} \frac{d u}{u}=\int_{0}^{\infty}|\hat{\psi}(-u)|^{2} \frac{d u}{u}=1
$$

(in such a case, $\psi$ is in general taken to be a real-valued function). If $\hat{\psi}$ is say differentiable, equation (1) basically means that $\hat{\psi}(0)=0$, otherwise stated:

$$
\int_{-\infty}^{\infty} \psi(x) d x=0
$$

Such a mother wavelet provides the following analysis of $L^{2}(I R)$ : for any $(b, a) \in$ $I R \times \mathbb{R}_{+}^{*}$, one introduces the wavelet:

$$
\psi_{(b, a)}(x)=\frac{1}{a} \psi\left(\frac{x-b}{a}\right)
$$

and one has the following representation theorem, the proof of which is well known and can be found for instance in [3] or [2].

Theorem 2.1 (CALderón). Let $\psi$ be a mother wavelet. Then any $f \in L^{2}(I R)$ decomposes as follows:

$$
\begin{aligned}
& f=\int_{\mathbb{R} \times \mathbb{R}_{+}^{*}} T_{f}(b, a) \psi_{(b, a)} \frac{d b d a}{a} \\
& T_{f}(b, a)=\left\langle f, \psi_{(b, a)}\right\rangle
\end{aligned}
$$

strongly in $L^{2}(I R) . \quad T_{f} \in L^{2}\left(I R \times \mathbb{R}_{+}^{*}\right)$ is called the wavelet transform of $f$ with respect to the analyzing wavelet $\psi$. If $\psi$ is sufficiently well localized in time and frequency (i.e. both $\psi$ and $\hat{\psi}$ have sufficient decay at infinity), $T_{f}$ gives informations on the time-frequency localization of $f$. Conversely, equation (4) states that the wavelet transform is invertible on its range, allowing the reconstruction of the analyzed function from its wavelet transform. 
If one restricts to the Hardy space $H^{2}(I R)$, a weaker admissibility condition (concerning only the positive frequency part of $\psi$ ) is sufficient. Simply assuming that:

$$
c_{\psi}=\int_{0}^{\infty}|\hat{\psi}(u)|^{2} \frac{d u}{u}=1
$$

Theorem 2.1 holds for any $f \in H^{2}(I R)$.

Let then $\psi(x)$ be an infinitesimal wavelet, and let $\Phi(x)$ be such that

$$
|\hat{\Phi}(\xi)|^{2}=\int_{|\xi|}^{\infty}|\hat{\psi}(u \operatorname{sgn}(\xi))|^{2} \frac{d u}{u} .
$$

In other words, $|\hat{\psi}(u \xi)|^{2}=-u \partial_{u}|\hat{\Phi}(u \xi)|^{2}$ for all $\xi \in \mathbb{R}$, and $\lim _{\xi \rightarrow \infty}|\hat{\Phi}(\xi)|^{2}=0$. $\Phi$ is called a scaling function, and one associates to it the corresponding:

$$
\Phi_{(b, a)}(x)=\frac{1}{a} \Phi\left(\frac{x-b}{a}\right) .
$$

To any $f \in L^{2}(I R)$ associate its smoothing (with respect to $\Phi$ ) at scale $a$ :

$$
s_{a}(x)=\int_{\mathbb{R}}\left\langle f, \Phi_{(b, a)}\right\rangle \Phi_{(b, a)}(x) d b
$$

that is:

$$
s_{a}(x)=\int_{a}^{\infty} d_{u}(x) \frac{d u}{u} .
$$

where $d_{a}(x)$ stands for the details of $f(x)$ at scale $a$ :

$$
d_{a}(x)=\int_{\mathbb{R}}\left\langle f, \psi_{(b, a)}\right\rangle \psi_{(b, a)}(x) d b
$$

Then $s_{a} \in L^{2}(\mathbb{R})$, and one has the following decompositions, whose proofs are immediate from that of theorem 2.1:

COROLlary 2.2. Let $\psi$ an infinitesimal wavelet, and $\Phi$ an associated scaling function. Then any $f \in L^{2}(\mathbb{R})$ can be expressed as:

$$
f=\lim _{a \rightarrow 0} s_{a}=s_{a_{0}}+\int_{0}^{a_{0}} d_{a} \frac{d a}{a}
$$

(for any $a_{0} \in \mathbb{R}_{+}^{*}$ ) strongly in $L^{2}(\mathbb{R})$. The corollary also holds in the $H^{2}(\mathbb{R})$ context. Let us now set

$$
D_{j}(x)=\int_{2^{-j-1}}^{2^{-j}} d_{a}(x) \frac{d a}{a} .
$$

Then $D_{j} \in L^{2}(\mathbb{R})$ represent the details of $f(x)$ visible at scale $2^{j+1}$ and not at scale $2^{j}$, and

$$
\widehat{D_{j}}(\xi)=\hat{f}(\xi) \int_{2^{-j-1}}^{2^{-j}}|\hat{\psi}(a \xi)|^{2} \frac{d a}{a} \text { a.e. }
$$


Introducing the function $\Psi(x)$, such that:

$$
|\widehat{\Psi}(\xi)|^{2}=\int_{\frac{1}{2}}^{1}|\hat{\psi}(a \xi)|^{2} \frac{d a}{a}
$$

one then has:

$$
\widehat{D_{j}}(\xi)=\hat{f}(\xi)\left|\widehat{\Psi}\left(2^{-j} \xi\right)\right|^{2}
$$

We will refer to the $\Psi(x)$ function as the (global or integrated) wavelet. Notice that equation (14) does not completely define the wavelet $\Psi$. Once again, one can restrict to wavelets with positive-valued Fourier transform, but this is not necessary. By construction, the integrated wavelets lead to a partition of unity in the Fourier space as follows:

$$
\sum_{j=-\infty}^{+\infty}\left|\widehat{\Psi}\left(2^{j} \xi\right)\right|^{2}=\left|\widehat{\Phi}\left(2^{j_{0}} \xi\right)\right|^{2}+\sum_{j=-\infty}^{j_{0}}\left|\widehat{\Psi}\left(2^{j} \xi\right)\right|^{2}=1
$$

for all $\xi \in \mathbb{R}$. We also have:

$$
|\widehat{\Psi}(\xi)|^{2}=|\hat{\Phi}(\xi / 2)|^{2}-|\hat{\Phi}(\xi)|^{2} .
$$

Defining the dilates and translates of the $\Phi^{j}$ and $\Psi^{j}$ as

$$
\begin{aligned}
& \Phi_{b}^{j}(x)=2^{-j} \Phi\left(2^{-j}(x-b)\right) \\
& \Psi_{b}^{j}(x)=2^{-j} \Psi\left(2^{-j}(x-b)\right)
\end{aligned}
$$

we then have:

THEOREM 2.3. Let $\psi$ an infinitesimal wavelet, and let $\Psi$ and $\Phi$ be associated integrated wavelet and scaling functions as in equations (14) and (6). Then any $f \in L^{2}(I R)$ can be decomposed as:

$$
f=\int_{\mathbb{R}}\left\langle f, \Phi_{b}^{j_{0}}\right\rangle \Phi_{b}^{j_{0}} d b+\sum_{j=-\infty}^{j_{0}} \int_{\mathbb{R}}\left\langle f, \Psi_{b}^{j}\right\rangle \Psi_{b}^{j} d b
$$

strongly in $L^{2}(I R)$.

We shall use the following notations

$$
\begin{aligned}
& T_{j} f(b)=\left\langle f, \Psi_{b}^{j}\right\rangle \\
& S_{j} f(b)=\left\langle f, \Phi_{b}^{j}\right\rangle
\end{aligned}
$$

2.2. The linear scheme. It is well known that the reconstructing and the analyzing wavelets can be decoupled i.e. one can use different infinitesimal wavelets for the computation of the coefficients and for the reconstruction of the analyzed function from the coefficients. In such a case, the admissibility condition (1) has to be modified accordingly.

A particular example of such a decoupling, which has been known for a long time, consists in taking formally a Dirac distribution for the reconstructing wavelet. Assuming instead of equation (1) that

$$
k_{\psi}=\int_{0}^{\infty} \hat{\psi}(u) \frac{d u}{u}=\int_{0}^{\infty} \hat{\psi}(-u) \frac{d u}{u}=1,
$$


one has the following decomposition of any $f \in L^{2}(I R)$ :

$$
f(x)=\int_{\mathbb{R}_{+}^{*}}\left\langle f, \psi_{(x, a)}\right\rangle \frac{d a}{a}
$$

strongly in $L^{2}(I R)$. This is the so-called Morlet reconstruction formula of $f$ from its wavelet coefficients. Such a linear analysis (linear in the $\psi$ function) generates a continuous multiresolution analysis as follows: introduce the linear scaling function $\varphi \in L^{1}(I R)$, defined by:

$$
\hat{\varphi}(\xi)=\int_{|\xi|}^{\infty} \hat{\psi}(u \operatorname{sgn}(\xi)) \frac{d u}{u} .
$$

$\varphi$ is also such that $\hat{\psi}(u \xi)=-u \partial_{u} \hat{\varphi}(u \xi)$ for all $\xi \in \mathbb{R}$. Associate to $\varphi$ the following functions:

$$
\varphi_{(b, a)}(x)=\frac{1}{a} \varphi\left(\frac{x-b}{a}\right) .
$$

Finally introduce:

$$
\delta_{a}(x)=T_{f}(x, a)=\left\langle f, \psi_{(x, a)}\right\rangle
$$

and

$$
\sigma_{a}(x)=\left\langle f, \varphi_{(x, a)}\right\rangle .
$$

One then has the linear analogue of Theorem 2.1 and the corresponding corollary:

THEOREM 2.4. Let $\psi \in L^{1}(I R)$ be a mother wavelet, such that equation (21) holds, and let $\varphi$ be

the associated linear scaling function. Then any $f \in L^{2}(I R)$ can be decomposed as:

$$
\begin{aligned}
f & =\lim _{a \rightarrow 0} \sigma_{a} \\
& =\sigma_{a_{0}}+\int_{0}^{a_{0}} \delta_{a} \frac{d a}{a} \quad, a_{0} \in \mathbb{R}_{+}^{*} \\
& =\int_{0}^{\infty} \delta_{a} \frac{d a}{a}
\end{aligned}
$$

strongly in $L^{2}(\mathbb{R})$.

The integrated wavelets are then defined as:

$$
\widehat{\Theta}(\xi)=\int_{\frac{1}{2}}^{1} \hat{\psi}(a \xi) \frac{d a}{a}
$$

and yield a partition of unity in the Fourier space:

$$
\sum_{j=-\infty}^{+\infty} \widehat{\Theta}\left(2^{j} \xi\right)=\hat{\varphi}\left(2^{j_{0}} \xi\right)+\sum_{j \leq j_{0}} \widehat{\Theta}\left(2^{j} \xi\right)=1
$$

The linear wavelets still appear as differences of smoothings at two consecutive scales, as:

$$
\widehat{\Theta}(\xi)=\hat{\varphi}(\xi / 2)-\hat{\varphi}(\xi)
$$


and every $f \in L^{2}(I R)$ decomposes as:

$$
f(x)=\sum_{j=-\infty}^{\infty}\left\langle f, \Theta_{x}^{j}\right\rangle=\left\langle f, \varphi_{x}^{j_{0}}\right\rangle+\sum_{j \leq j_{0}}\left\langle f, \Theta_{x}^{j}\right\rangle
$$

where $\Theta_{b}^{j}(x)=\Theta^{j}(x-b)$.

\section{Associated approximate filters.}

3.1. Pyramidal algorithms. Let us recall the usual algorithmic structure associated with a multiresolution analysis. Let $\phi$ and $\psi$ be respectively a scaling function and a wavelet associated with the multiresolution analysis, and set, $\forall f \in L^{2}(\mathbb{R})$

$$
\begin{aligned}
& T_{j} f(n)=\left\langle f, \psi_{j n}\right\rangle=2^{-j} \int_{\mathbb{R}} f(x) \psi\left(2^{-j}(x-n)\right)^{*} d x \\
& S_{j} f(n)=\left\langle f, \phi_{j n}\right\rangle=2^{-j} \int_{\mathbb{R}} f(x) \phi\left(2^{-j}(x-n)\right)^{*} d x
\end{aligned}
$$

Notice that for any value of the scale parameter $a=2^{j}$, we sample the corresponding wavelet and scaling function transform at unit sampling frequency.

Then if $\psi$ and $\phi$ are related by

$$
\begin{aligned}
& \hat{\phi}(2 \xi)=m_{0}(\xi) \hat{\phi}(\xi) \\
& \hat{\psi}(2 \xi)=m_{1}(\xi) \hat{\phi}(\xi)
\end{aligned}
$$

where $m_{0}$ and $m_{1}$ are the $2 \pi$-periodic low-pass and high-pass filters

$$
\begin{aligned}
& m_{0}(\xi)=\sum_{k} h_{k} e^{i k \xi} \\
& m_{1}(\xi)=\sum_{k} g_{k} e^{i k \xi}
\end{aligned}
$$

then the coefficients may be computed using the following pyramidal algorithm

$$
\begin{aligned}
& T_{j} f(n)=\sum_{k} g_{k}^{*} S_{j-1} f\left(n-k 2^{-j-1}\right) \\
& S_{j} f(n)=\sum_{k} h_{k}^{*} S_{j-1} f\left(n-k 2^{-j-1}\right)
\end{aligned}
$$

The algorithm is called pyramidal since scaled copies of the same filters are used throughout the calculation, and the coefficients are obtained by successive convolutions with such filters. It is easy to see that the total number of multiplications necessary to process $N$ samples of say $S_{0} f$ is proportional to $N \log (N)$. It is schematically described in figure 1 (in the particular situation where the $m_{0}(\xi)$ filter has only 3 non vanishing coefficients).

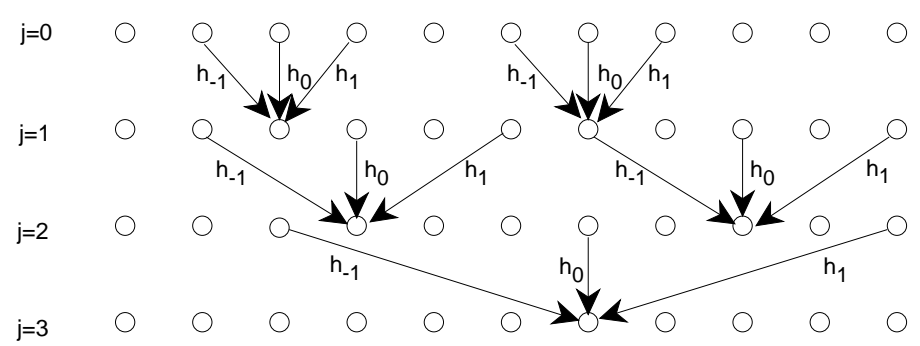

Figure 1: QMF algorithm associated with the wavelet transform on a fine grid 
3.2. Approximate filters. We address now the problem of discretization of the previous wavelet decompositions. Up to now, we have only obtained decompositions that are discrete with respect to the scale, and continuous with respect to the position. The problem is that no discrete filters are "a-priori" available.

We shall work with both the linear and the bilinear analysis-reconstruction schemes at the beginning, and specify our choice later on. We shall assume from now on that a pair of functions

$$
\begin{aligned}
& m_{0}(\xi)=\frac{\widehat{\Phi}(2 \xi)}{\widehat{\Phi}(\xi)} \\
& m_{1}(\xi)=\frac{\widehat{\Psi}(2 \xi)}{\widehat{\Phi}(\xi)}
\end{aligned}
$$

can be defined almost everywhere in $I R$. This is clearly the case in the bilinear scheme, where $|\widehat{\Phi}|$ is monotonic for both $\xi \geq 0$ and $\xi \leq 0$. The problem is that in general, such a $m_{0}$-function is not $2 \pi$-periodic, and thus cannot be used in a pyramidal algorithm.

Nevertheless, a modification is possible. Indeed, if $\widehat{\Phi}(\xi)$ is "concentrated" around the origin, in say the interval $[-\pi, \pi]^{1}$, then one may expect that $\widehat{\Phi}$ "does not see too much" the non-periodicity of $m_{0}(\xi)$, and that $m_{0}(\xi) \widehat{\Phi}(\xi)$ can be well approximated by $m_{0}^{a}(\xi) \widehat{\Phi}(\xi)$, for some $2 \pi$-periodic function $m_{0}^{a}(\xi)^{2}$.

Let us introduce here for convenience the following subspace of $L^{2}(I R)$ :

$$
\mathcal{U}_{0}=\left\{f \in L^{2}(\mathbb{R}), f=\sum \alpha_{k} \Phi(x-k),\left\{\alpha_{k}\right\} \in \ell^{2}(\mathbb{Z})\right\}
$$

We will assume that the collection $\{\Phi(x-k), k \in \mathbb{Z}\}$ is a Riesz basis of $\mathcal{U}_{0}$, or, equivalently, there exists two finite and nonzero constants $A$ and $B$ such that

$$
A \leq \sum_{k}|\widehat{\Phi}(\xi+2 \pi k)|^{2} \leq B \text { almost everywhere }
$$

Then it follows from general results that there exists a function $\chi \in L^{2}(\mathbb{R})$ such that the sequence $\{\chi(x-k), k \in \mathbb{Z}\}$ is the basis of $\left.\mathcal{U}_{0}\right)$ biorthogonal to $\{\Phi(x-k), k \in \mathbb{Z}\}$. $\chi$ is given by its Fourier transform

$$
\widehat{\chi}(\xi)=\frac{\widehat{\Phi}(\xi)}{\sum_{k} \mid \widehat{\Phi}(\xi+2 \pi k)^{2}}
$$

Consider now the discretization of the functions $T_{j} f(x)$ and $S_{j} f(x)$, that we shall denote by $T_{j}^{d} f(n)$ and $S_{j}^{d} f(n)$ respectively:

$$
\begin{aligned}
& S_{j}^{d} f(n)=S_{j} f(n) \forall n \in \mathbb{Z} \\
& T_{j}^{d} f(n)=T_{j} f(n) \forall n \in \mathbb{Z}
\end{aligned}
$$

\footnotetext{
1 This assumption is motivated by the fact that we will sample the wavelet transform and the scaling function transform at unit sampling frequency

2 A natural candidate for $m_{0}^{a}(\xi)$ is the periodization $\sum_{k} m_{0}(\xi+2 \pi k)$ of $m_{0}(\xi)$, but as we shall see, there are many other choices.
} 


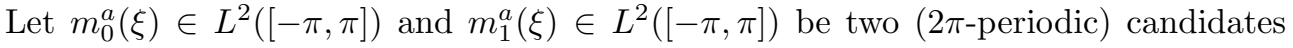
for approximate filters, and denote by $\left\{h_{k}^{a}, k \in \mathbb{Z}\right\}$ and $\left\{g_{k}^{a}, k \in \mathbb{Z}\right\}$ their respective Fourier coefficients. We will then set:

$$
\left\{\begin{aligned}
T_{1}^{a} f(n) & =\sum_{k} g_{k}^{a *} S_{0}^{d} f(n-k) \\
S_{1}^{a} f(n) & =\sum_{k} h_{k}^{a *} S_{0}^{d} f(n-k)
\end{aligned}\right.
$$

and for $j>1$

$$
\left\{\begin{aligned}
T_{j}^{a} f(n) & =\sum_{k} g_{k}^{a *} S_{j-1}^{a} f\left(n-2^{j-1} k\right) \\
S_{j}^{a} f(n) & =\sum_{k} h_{k}^{a *} S_{j-1}^{a} f\left(n-2^{j-1} k\right)
\end{aligned}\right.
$$

Our purpose is to compare such "algorithmic expressions" ${ }^{3}$ with the exact expressions $S_{j}^{d} f$ and $T_{j}^{d} f$, and to find "best approximants" for the $m_{0}^{a}$ and $m_{1}^{a}$ filters.

The first remark is the following

PROPOSITION 3.1.

1. $\operatorname{Ker}\left(S_{0}^{d}\right)=\mathcal{U}_{0}^{\perp}$.

2. $S_{j}^{a} \cdot \mathcal{U}_{0}^{\perp}=T_{j}^{a} \cdot \mathcal{U}_{0}^{\perp}=0$ For any $j=1, \ldots$.

Proof: The first part is a direct consequence of the definition of $\mathcal{U}_{0}$, and implies the second part by definition (43).

Our main result is the following.

THEOREM 3.2. Let $\Phi(x)$ and $\Psi(x)$ be the scaling function and the integrated wavelet respectively associated with the infinitesimal wavelet $\psi(x)$, and let $m_{0}(\xi)$ and $m_{1}(\xi)$ be the associated low-pass and high-pass filters. For $i=0,1$ set

$$
\mu\left(m_{i}, m_{i}^{a}\right)=\left[\int_{\mathbb{R}}\left|\left(m_{i}^{a}(\xi)-m_{i}(\xi)\right) \widehat{\Phi}(\xi)\right|^{2} d \xi\right]^{1 / 2}
$$

Then the following properties are satisfied

1. There exists a unique pair of $2 \pi$-periodic filters $m_{i}^{a}(\xi)=\tilde{m}_{i}(\xi)$ minimizing $\mu\left(m_{i}, m_{i}^{a}\right)$, given by:

$$
\begin{gathered}
\tilde{m}_{0}(\xi)=\frac{\sum_{k \in \boldsymbol{Z}} \widehat{\Phi}(\xi+2 \pi k)^{*} \widehat{\Phi}(2(\xi+2 \pi k))}{\sum_{k \in \boldsymbol{Z}}|\widehat{\Phi}(\xi+2 \pi k)|^{2}} \\
\tilde{m}_{1}(\xi)=\frac{\sum_{k \in \boldsymbol{Z}} \widehat{\Phi}(\xi+2 \pi k)^{*} \widehat{\Psi}(2(\xi+2 \pi k))}{\sum_{k \in \boldsymbol{Z}}|\widehat{\Phi}(\xi+2 \pi k)|^{2}}
\end{gathered}
$$

2. For the above choice of filters, and setting

$$
C_{i}=\operatorname{ess} \sup _{\xi \in \mathbb{R}}\left|\tilde{m}_{i}(\xi)\right|, \quad i=0,1
$$

the following inequalities hold:

$$
\left\|S_{j}^{a} f-S_{j}^{d} f\right\|_{\infty} \leq \mu\left(\tilde{m}_{0}, m_{0}\right) 2^{\frac{1-j}{2}} \frac{1-\left(C_{0} \sqrt{2}\right)^{j}}{1-C_{0} \sqrt{2}}\|f\|_{2}
$$

(49) $\left\|T_{j}^{a} f-T_{j}^{d} f\right\|_{\infty} \leq 2^{\frac{1-j}{2}}\left(\mu\left(\tilde{m}_{1}, m_{1}\right)+C_{1} \mu\left(\tilde{m}_{0}, m_{0}\right) \sqrt{2} \frac{1-\left(C_{0} \sqrt{2}\right)^{j-1}}{1-C_{0} \sqrt{2}}\right)\|f\|_{2}$

\footnotetext{
${ }^{3}$ because this is precisely what is numerically computed in practice
} 
3. For any $f \in \mathcal{U}_{0}$

$$
\begin{aligned}
& S_{1}^{a} f=S_{1}^{d} f \\
& T_{1}^{a} f=T_{1}^{d} f
\end{aligned}
$$

Before giving the proof of the theorem, let us give the following immediate corollary:

Corollary 3.3. Assume that the infinitesimal wavelet $\psi(x)$ is associated with a linear "analysis-reconstruction" scheme. Then the associated approximate QMFs $\tilde{m}_{i}(\xi)$ given by equations (45) and (46) satisfy

$$
\tilde{m}_{0}(\xi)+\tilde{m}_{1}(\xi)=1
$$

so that we have the "reconstruction algorithm"

$$
S_{0} f(n)=\sum_{j \geq 0} T_{j}^{a}(n)
$$

This reconstruction formula is the discrete counterpart of Morlet's reconstruction formula (22). It was also obtained in a slightly different context by Beylkin and Saito [5].

Proof of the theorem: Using the inequality $\|f\|_{\infty} \leq\|\hat{f}\|_{1} / 2 \pi$, we shall directly work in the Fourier space. First of all, we clearly have

$$
\begin{aligned}
\left\|\widehat{S_{1}^{a} f}-\widehat{S_{1}^{d} f}\right\|_{1} & \leq \sum_{k \in \mathbb{Z}} \int_{0}^{2 \pi}\left|\left[m_{0}^{a}(\xi)-m_{0}(\xi+2 \pi k)\right] \widehat{\Phi}(\xi+2 \pi k) \hat{f}(\xi+2 \pi k)\right| d \xi \\
& \leq \int_{\mathbb{R}}\left|\left[m_{0}^{a}(\xi)-m_{0}(\xi)\right] \widehat{\Phi}(\xi) \hat{f}(\xi)\right| d \xi \\
& \leq 2 \pi\|f\|_{2}\left[\int_{\mathbb{R}}\left[m_{0}^{a}(\xi) \widehat{\Phi}(\xi)-\left.\widehat{\Phi}(2 \xi)\right|^{2} d \xi\right]^{1 / 2}=2 \pi \mu\left(m_{0}^{a}, m_{0}\right)\|f\|_{2}\right.
\end{aligned}
$$

(the last inequality coming from the Cauchy-Schwartz inequality). This explains the occurence of such a term in our formulation. The minimization of this term is a classical problem, and leads to

$$
\int_{\mathbb{R}} \Phi(x+k)^{*}\left[\sum_{l} h_{l}^{a} \Phi(x+l)-\frac{1}{2} \Phi\left(\frac{x}{2}\right)\right] d x=0 \quad \forall k \in \mathbb{Z}
$$

or, otherwise stated

$\left(55 \int_{0}^{2 \pi} e^{i k \xi}\left[m_{0}^{a}(\xi) \sum_{l \in \mathbb{Z}}|\widehat{\Phi}(\xi+2 \pi l)|^{2}-\sum_{l \in \mathbb{Z}} \widehat{\Phi}(\xi+2 \pi l)^{*} \widehat{\Phi}(2(\xi+2 \pi l))\right] d \xi=0 \quad \forall k \in \mathbb{Z}\right.$

The unique solution is precisely that given in equation (45). The estimation of $\left\|T_{1}^{a} f-T_{1}^{d} f\right\|_{\infty}$ is completely similar, and leads to the approximate filter given in equation (45). The details are left to the reader.

Let us now consider larger scales. Before going into the details, let us introduce for convenience the following "intermediate" sequences:

$$
\left\{\begin{array}{c}
T_{j}^{i} f(n)=\sum_{k} g_{k}^{a *} S_{j-1}^{d} f\left(n-2^{j-1} k\right) \\
S_{j}^{i} f(n)=\sum_{k} h_{k}^{a *} S_{j-1}^{d} f\left(n-2^{j-1} k\right)
\end{array}\right.
$$

Then clearly

$$
\begin{aligned}
& \left\|\widehat{S_{j}^{a} f}-\widehat{S_{j}^{d} f}\right\|_{1} \leq\left\|\widehat{S_{j}^{a} f}-\widehat{S_{j}^{i} f}\right\|_{1}+\left\|\widehat{S_{j}^{i} f}-\widehat{S_{j}^{d} f}\right\|_{1} \\
& \left\|\widehat{T_{j}^{a} f}-\widehat{T_{j}^{d} f}\right\|_{1} \leq\left\|\widehat{T_{j}^{a} f}-\widehat{T_{j}^{i} f}\right\|_{1}+\left\|\widehat{T_{j}^{i} f}-\widehat{T_{j}^{d} f}\right\|_{1}
\end{aligned}
$$


Again we focus on the approximations $S_{j}^{a} f$, the proof for the details $T_{j}^{a} f$ being completely similar.

$$
\begin{aligned}
\left\|\widehat{S_{j}^{i} f}-\widehat{S_{j}^{d} f}\right\|_{1}= & \int_{0}^{2 \pi} \mid \sum_{k \in \mathbb{Z}} m_{0}\left(2^{j-1}(\xi+2 \pi k)\right) \widehat{\Phi}\left(2^{j-1}(\xi+2 \pi k)\right) \hat{f}(\xi+2 \pi k) \\
& \quad-\sum_{k \in \mathbb{Z}} m_{0}^{a}\left(2^{j-1} \xi\right) \widehat{\Phi}\left(2^{j-1}(\xi+2 \pi k)\right) \hat{f}(\xi+2 \pi k) \mid d \xi \\
2 \pi \leq\|f\|_{2}\left[\int_{\mathbb{R}}\left|m_{0}^{a}\left(2^{j-1} \xi\right) \widehat{\Phi}\left(2^{j-1} \xi\right)-\widehat{\Phi}\left(2^{j} \xi\right)\right|^{2} d \xi\right]^{1 / 2} & \\
\leq & 2 \pi\|f\|_{2} 2^{\frac{1-j}{2}}\left[\int_{\mathbb{R}}\left|m_{0}^{a}(\xi) \widehat{\Phi}(\xi)-\widehat{\Phi}(2 \xi)\right|^{2} d \xi\right]^{\frac{1}{2}}=2 \pi 2^{\frac{1-j}{2}} \mu\left(m_{0}^{a}, m_{0}\right)\|f\|_{2}
\end{aligned}
$$

(58)

The second term is estimated as follows

$$
\begin{aligned}
\left\|\widehat{S_{j}^{a} f}-\widehat{S_{j}^{i} f}\right\|_{1} & \leq \int_{\mathbb{R}}\left|m_{0}^{a}\left(2^{j-1} \xi\right)\right|\left|\widehat{S_{j-1}^{a}} f(\xi)-\widehat{S_{j-1}^{d}} f(\xi)\right| d \xi \\
& \leq \operatorname{ess} \sup _{\xi \in \mathbb{R}}\left|m_{0}^{a}\right|\left\|\widehat{S_{j-1}^{a} f} f-\widehat{S_{j-1}^{d} f}\right\|_{1}
\end{aligned}
$$

Summarizing, for $m_{0}^{a}(\xi)=\tilde{m}_{0}(\xi)$ we have

$$
\begin{aligned}
\left\|\widehat{S_{j}^{a} f}-\widehat{S_{j}^{d} f}\right\|_{1} & \leq 2 \pi 2^{\frac{1-j}{2}} \mu\left(\tilde{m}_{0}, m_{0}\right)\|f\|_{2}+C_{0}\left\|\widehat{S_{j-1}^{a} f}-\widehat{S_{j-1}^{d}} f\right\|_{1} \\
& \leq 2 \pi 2^{\frac{1-j}{2}} \mu\left(\tilde{m}_{0}, m_{0}\right)\left(1+C_{0} \sqrt{2}+\left(C_{0} \sqrt{2}\right)^{2}+\ldots+\left(C_{0} \sqrt{2}\right)^{j-1}\right)\|f\|_{2} \\
& \leq 2 \pi 2^{\frac{1-j}{2}} \mu\left(\tilde{m}_{0}, m_{0}\right) \frac{1-\left(C_{0} \sqrt{2}\right)^{j}}{1-C_{0} \sqrt{2}}\|f\|_{2}
\end{aligned}
$$

The same kind of estimates yields the error estimate for the $T_{j}^{a} f$ coefficients. This achieves the proof of the two first items of the theorem.

Let us turn to the third part of the theorem. Let us then assume that $f \in \mathcal{U}_{0}$. This means that in the Fourier space, $f$ is of the form

$$
\hat{f}(\xi)=F(\xi) \widehat{\Phi}(\xi)
$$

for some $2 \pi$-periodic function $F \in L^{2}([-\pi, \pi])$. Then an explicit computation of $\widehat{S_{1}^{a} f}-\widehat{S_{1}^{d} f}$ yields

$$
\begin{aligned}
\widehat{S_{1}^{a f}}-\widehat{S_{1}^{d} f} & =\sum_{k \in \mathbb{Z}}\left(m_{0}^{a}(\xi)^{*}-m_{0}(\xi+2 \pi k)^{*}\right)|\widehat{\Phi}(\xi+2 \pi k)|^{2} F(\xi) \\
& =0 \text { if } m_{0}^{a}=\tilde{m}_{0} .
\end{aligned}
$$

This concludes the proof of the theorem.

Remark: asymptotic behaviour:

It is interesting to see what is the asymptotic behaviour of the estimates when $j \rightarrow \infty$. Consider for instance the estimate of $\left\|S_{j}^{a} f-S_{j}^{d} f\right\|_{\infty}$, the coefficient of $\|f\|_{2}$ goes as:

$$
\left\|S_{j}^{a} f-S_{j}^{d} f\right\|_{\infty} \sim_{j \rightarrow \infty} \mu\left(\tilde{m}_{0}, m_{0}\right) \sqrt{2} C_{0}^{j} /\left(C_{0} \sqrt{2}-1\right)\|f\|_{2}
$$

(for $C_{0} \sqrt{2} \neq 1$ ) in the limit. The limit is finite for $C_{0}=1$ and zero for $C_{0}<1$, while it diverges for $C_{0}>1$. In the two first cases, this means that the accumulation of errors due to the approximate algorithm is compensated by the fact that $S_{j} f$, lying at larger and larger scales, is all the time sampled at the same frequency. This shows that "redundancy implies stability". 
3.3. Decay of approximate filter coefficients. The localization properties of the $\left\{h_{k}\right\}$ (and thus $\left\{g_{k}\right\}$ ) approximate filters can be directly related to the regularity properties of the scaling function, as follows.

THEOREM 3.4. Let $\phi \in L^{1}(I R)$ be a p-times differentiable scaling function, and let $\tilde{m}_{0}(\xi)=\sum_{k} h_{k} e^{i k \xi}$ be the low-pass filter defined according to equation (45). Then if for any $m=0,1, \ldots p$

$$
\left|\frac{d^{m} \hat{\phi}(\xi)}{d \xi^{m}}\right| \leq \frac{K_{m}}{(1+|\xi|)^{\frac{1}{2}+\epsilon}}
$$

for some positive constants $K_{m}, \epsilon$, then

$$
h_{k}=O\left(k^{-p}\right)
$$

Proof: Assume that $\hat{\phi}(\xi)$ is $p$ times differentiable. Then after $p$ differentiations, equation (45) yields

$$
\frac{d^{p} \tilde{m}_{0}(\xi)}{d \xi^{p}}=\frac{\sum_{k} G(\xi+2 \pi k)}{\left[\sum_{k}|\hat{\phi}(\xi+2 \pi k)|^{2}\right]^{p}}
$$

where $G(\xi)$ is a finite linear combination of terms of the form

$$
\frac{d^{m} \hat{\phi}(\xi)}{d \xi^{m}} \frac{d^{p-m} \hat{\phi}(\xi)^{*}}{d \xi^{p-m}}
$$

and

$$
\frac{d^{m} \hat{\phi}(\xi)}{d \xi^{m}} \frac{d^{p-m} \hat{\phi}(2 \xi)^{*}}{d \xi^{p-m}}
$$

and their complex conjugates. Then the estimate (66) gives

$$
\frac{d^{p} \tilde{m}_{0}(\xi)}{d \xi^{p}} \in L^{\infty}([0,2 \pi])
$$

and

$$
\frac{d^{p} \tilde{m}_{0}(\xi)}{d \xi^{p}} \in C(I R)
$$

Moreover,

$$
k^{p} h_{k}=\frac{i^{-p}}{2 \pi} \int_{0}^{2 \pi} \frac{d^{p} \tilde{m}_{0}(\xi)}{d \xi^{p}} e^{-i k \xi} d \xi
$$

leads to

$$
|k|^{p}\left|h_{k}\right| \leq \frac{1}{2 \pi} \int\left|\frac{d^{p} \tilde{m}_{0}(\xi)}{d \xi^{p}}\right| d \xi \leq\left\|\frac{d^{p} \tilde{m}_{0}}{d \xi^{p}}\right\|_{\infty}
$$

which proves the theorem.

Thus, under some weak assumptions on the scaling function, it is possible to get well localized filters. However, this problem is completely independent of the accuracy problem addressed in the previous section.

Notice also that theorem 3.4 is to be compared with similar results in the case of classical multiresolution analysis, which led to the notion of $r$-regular multiresolution analysis (see [6]). 
3.4. The bilinear scheme. We have seen in the corollary of the previous subsection that the approximate filters $\tilde{m}_{0}$ and $\tilde{m}_{1}$ given in (45) and (46) are ideally adapted to the linear analysis-reconstruction scheme. However, in the bilinear case, $\tilde{m}_{0}$ and $\tilde{m}_{1}$ cannot be directly used to "reconstruct" the analyzed function from the approximate coefficients, since they do not fulfill the QMF condition:

$$
\left|\tilde{m}_{0}(\xi)\right|^{2}+\left|\tilde{m}_{1}(\xi)\right|^{2} \neq 1 \text { in general }
$$

A possibility is then to use different filters for the reconstruction, for instance $\tilde{m}_{0}$ as low-pass filter, and

$$
\tilde{m}_{1}^{r}(\xi)=\frac{1-\left|\tilde{m}_{0}(\xi)\right|^{2}}{\tilde{m}_{1}(\xi)}
$$

as high-pass filter. One has in such a case to be careful with the zeros of the $\tilde{m}_{1}(\xi)$ filter.

As an alternative, the same kind of analysis as before can be performed in the bilinear "analysis-reconstruction" scheme. The previous arguments have to be applied to the details and approximations instead of the wavelet coefficients themselves.

$$
\widehat{s_{j}}(\xi)=\left|\widehat{\Phi}\left(2^{j} \xi\right)\right|^{2} \hat{f}(\xi)
$$

Using again approximate filters to evaluate the coefficients, one is then naturally led to the quantity:

$$
\widehat{s_{j}^{a}}(\xi)=\left|m_{0}^{a}(\xi)\right|^{2} \widehat{s_{j-1}^{a}} f(\xi)
$$

At the first step for instance, one has to evaluate

$$
\left\|s_{1}^{a}-s_{1}^{d}\right\|_{\infty} \leq\|f\|_{2} \int_{\mathbb{R}}\left|\left[\left|m_{0}^{a}(\xi)\right|^{2}-\left|m_{0}(\xi)\right|^{2}|\widehat{\Phi}(\xi)|^{2}\right]\right|^{2} d \xi
$$

The minimization of such a quantity naturally leads to

$$
\left|m_{0}^{a}(\xi)\right|^{2}=\frac{\sum_{k \in \mathbb{Z}}|\widehat{\Phi}(\xi+2 \pi k)|^{2}|\widehat{\Phi}(2 \xi+4 \pi k)|^{2}}{\sum_{k \in \mathbb{Z}}|\widehat{\Phi}(\xi+2 \pi k)|^{4}}
$$

and similarly

$$
\left|m_{1}^{a}(\xi)\right|^{2}=\frac{\sum_{k \in \mathbb{Z}}|\widehat{\Phi}(\xi+2 \pi k)|^{2}|\widehat{\Psi}(2 \xi+4 \pi k)|^{2}}{\sum_{k \in \mathbb{Z}}|\widehat{\Phi}(\xi+2 \pi k)|^{4}}
$$

It is worth noticing that in such a case, the bilinear scheme is well suited for this pair of filters, and ensures the validity of the usual QMF relation:

$$
\left|m_{0}^{a}(\xi)\right|^{2}+\left|m_{1}^{a}(\xi)\right|^{2}=1
$$

Moreover, it is easy to derive the "bilinear counterpart" of theorem 3.4, relating the length of the approximate filters to the regularity of the scaling function. 


\subsection{Some complementary remarks.}

1. The algorithm described above is actually adapted to the problem of finding approximate discretization of Littlewood-Paley decompositions, and is a priori independent of the linear or bilinear schemes derived from continuous wavelet decompositions. In other words, there is no connection between the $b$ discretization problem and the scale discretization (which is not a true discretization in the method reported in section 2). Corollary 3.3 simply states that if one considers the filters $\tilde{m}_{0}$ and $\tilde{m}_{1}$, the choice of the linear scheme yields simpler reconstruction formulas.

2. Throughout this paper, we have implicitely fixed a reference scale by the choice of a sampling frequency equal to one for all the voices of the wavelet transform. A change of this sampling frequency is equivalent to a global scaling of $L^{2}(I R)$.

3. Assume that we are in the case of a scaling function with exponential decay in the Fourier space (i.e. $\widehat{\Phi}(\xi) \leq C_{\phi} e^{-\alpha|\xi|}$ for some positive $\alpha$ ). Then it is not very difficult to show that (in the case of a unit sampling frequency) the approximation of the filters obtained by sampling the inverse Fourier transform of $m_{0}$ leads to an error on the scaling function coefficients of the order $e^{-\alpha \pi}$. In the same way, defining the approximate $2 \pi$-periodic high-pass filter by the QMF-relation leads to the same kind of error estimate for the wavelet coefficients.

4. Obviously, it follows from the expressions of the approximate filters (both in the linear and bilinear schemes) that if $\Phi$ and $\Psi$ are associated with an usual multiresolution analysis, with $2 \pi$-periodic filters, one recovers $m_{0}^{a}=m_{0}$ and $m_{1}^{a}=m_{1}$.

5. It was shown in [2] how to use Calderón's formula to get descriptions of the Fourier space different from the Littlewood-Paley one, by replacing the powers of 2 by an arbitrary monotonic sequence of scale parameters. It sounds reasonable to think of corresponding approximate algorithms similar to the one described above, at least for rational scale parameters. However this has not been done at the present time.

4. Examples. There are many examples of continuous wavelets for which an efficient algorithm is needed. We describe here some very simple examples (the filters coefficients have been computed using the Mathematica Package).

4.1. The LOG and DOG wavelets. The LOG wavelets are widely used in the context of computer vision. LOG stands for "Laplacian Of Gaussians". As stressed in [2], in the linear "decomposition-reconstruction" scheme, if

$$
\psi(x)=\frac{1}{\sqrt{\pi}}\left(1-x^{2}\right) e^{-x^{2} / 2}
$$

the associated scaling function and integrated wavelet are given by

$$
\varphi(x)=\frac{1}{2 \sqrt{2 \pi}} e^{-x^{2} / 2}
$$

and

$$
\Theta(x)=\frac{1}{2 \sqrt{2 \pi}}\left(e^{-x^{2} / 2}-2 e^{-2 x^{2}}\right)
$$

The integrated wavelet is then a DOG wavelet ("Difference Of Gaussians"), and there is no problem to derive the details coefficients $T_{j}^{d} f$ form the approximations $S_{j}^{d} f$. But one clearly need an efficient algorithm to compute the approximations. In general, the scaling function and wavelet have to be scaled properly in order that the corresponding 
transforms can be accurately sampled at unit sampling frequency. We shall then consider more general scaling functions

$$
\hat{\varphi}(\xi)=e^{-\xi^{2} / \alpha}
$$

with the corresponding integrated wavelets. We give as examples the plots of the approximate low-pass filters $\tilde{m}_{0}(\xi)$ (the high-pass filter $\tilde{m}_{1}(\xi)$ is easy to deduce), and the coefficients of $\tilde{m}_{0}(\xi)$ and $\tilde{m}_{1}(\xi)$ for $\alpha=4$ and $\alpha=6$ respectively. It is worth noticing that in both cases (and in fact for any positive $\alpha$ ), the $\left\{h_{k}\right\}$ and $\left\{g_{k}\right\}$ sequences are rapidly decreasing, as a consequence of theorem 3.4.

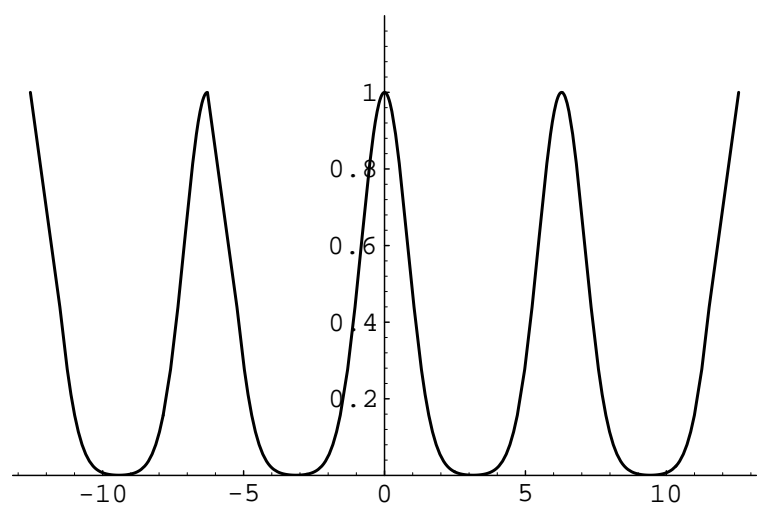

Figure 2: Approximate low-pass filter for the DOG wavelet with $\alpha=4$

4.1.1. $\alpha=4$. $h_{k}$ coefficients

$\{0.3256327400276189,0.23348983204217,0.085798244731082$,

$0.01625749135447543,0.0015489926732795,0.0000922406284932685$,

$$
\begin{aligned}
& -6.329410^{-6}, 5.0466410^{-6},-3.037210^{-6}, \\
& 1.8405310^{-6},-1.1159510^{-6}, 6.7677210^{-7}, \\
& \left.-4.1046310^{-7}, 2.4895410^{-7},-1.5099710^{-7}, 9.1584410^{-8}\right\}
\end{aligned}
$$

$g_{k}$ coefficients

$\{0.6743672599723812,-0.2334898320421699,-0.085798244731082$,

$-0.01625749135447542,-0.00154899267327945,-0.000092240628493343$,

(84) $6.329410^{-6},-5.0466410^{-6}, 3.037210^{-6}$,

$-1.8405310^{-6}, 1.1159510^{-6},-6.7677210^{-7}$,

$\left.4.1046310^{-7},-2.4895410^{-7}, 1.5099710^{-7},-9.1584410^{-8}\right\}$

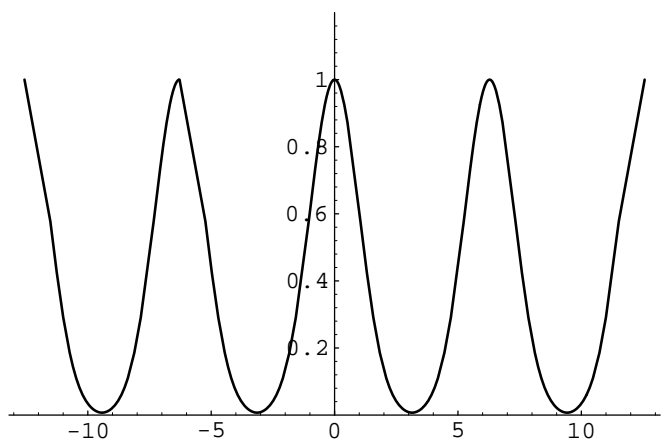

Figure 3: Approximate low-pass filter for the DOG wavelet with $\alpha=6$ 
4.1.2. $\alpha=6$. The corresponding $h_{k}$ coefficients read

$$
\begin{aligned}
& \{0.3972771041574456,0.2433136948393599,0.05323383400865474, \\
& 0.004788125238023543,-0.00002934485917262438, \\
& 0.00007751303939803943,-0.00003576989742401233, \\
& 0.00001688424764446744,-7.9739410^{-6}, \\
& 3.7664510^{-6},-1.7791310^{-6}, 8.4039810^{-7}, \\
& \left.-3.9697610^{-7}, 1.8751810^{-7},-8.8577310^{-8}, 4.1840910^{-8}\right\}
\end{aligned}
$$

and the $g_{k}$ coefficients are

$$
\begin{aligned}
& \{0.6027228958425545,-0.2433136948393598,-0.05323383400865478, \\
& -0.004788125238023527,0.00002934485917263695, \\
& -0.00007751303939808579,0.00003576989742395847, \\
& -0.00001688424764445047,7.9739410^{-6}, \\
& -3.7664510^{-6}, 1.7791310^{-6},-8.4039810^{-7}, \\
& \left.3.9697610^{-7},-1.8751810^{-7}, 8.8577310^{-8},-4.1840910^{-8}\right\}
\end{aligned}
$$

4.1.3. Precision of the algorithm:. As we have seen, the estimate of the accuracy of the approximate algorithm is governed by the functional $\mu\left(m_{0}, \tilde{m}_{0}\right)$. We present here numerical estimation of this quantity for the DOG wavelets, for various values of the $\alpha$ parameter. For instance, $\mu\left(m_{0}, \tilde{m}_{0}\right)=1.0363210^{-17}$ for $\alpha=1$, $\mu\left(m_{0}, \tilde{m}_{0}\right)=1.6390910^{-6}$ for $\alpha=3$ and $\mu\left(m_{0}, \tilde{m}_{0}\right)=0.0012859$ for $\alpha=6$. Figure 4 represents the logarithm of $\mu\left(m_{0}, \tilde{m}_{0}\right)$ as a function of $\alpha$.

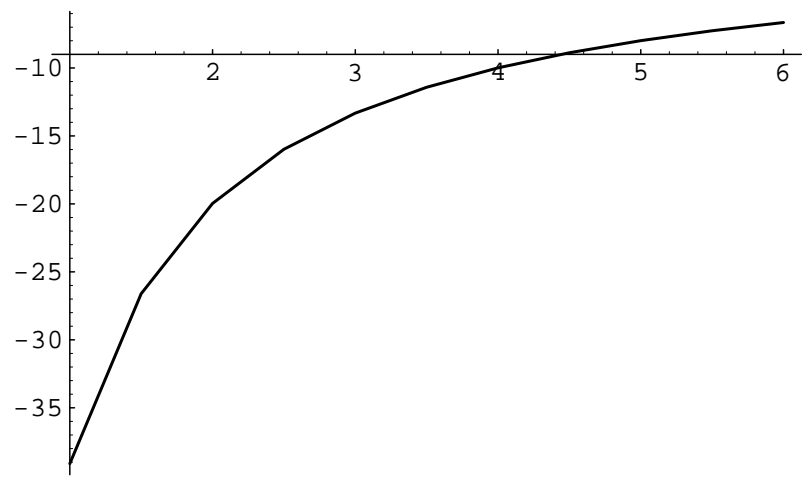

Figure 4: Logarithm of $\mu\left(m_{0}, \tilde{m}_{0}\right)$ as a function of $\alpha$

4.2. "Exponential-type" wavelets. These wavelets are real-valued wavelets, characterized by their exponential decay in the Fourier space. Let

$$
\hat{\psi}_{n}(\xi)=\frac{1}{(n-1) !} \frac{|\xi|^{n}}{\alpha^{n}} e^{-|\xi| / \alpha}
$$

$n=1, \ldots \infty$ then controls the number of vanishing moments, and $\hat{\psi}_{n}(\xi)$ has exponential decay for all $n$. A direct computation yields the corresponding scaling function

$$
\hat{\varphi}_{n}(\xi)=e^{-|\xi| / \alpha} \sum_{p=0}^{n-1} \frac{1}{p !} \frac{|\xi|^{p}}{\alpha^{p}}
$$

Notice that $\hat{\phi}_{n}(\xi) \sim_{\xi \sim 0} 1+O\left(|\xi|^{n}\right)$. Then $\hat{\phi}_{n} \in C^{n-1}(\mathbb{R})$ and $\left|d^{m} \hat{\phi} / d \xi^{m}\right|$ has exponential decay $\forall m=0, \ldots n-1$, which implies that $h_{k}=O\left(k^{1-n}\right)$. 
The integrated wavelets are easy to deduce, and the associated low-pass filter is represented in figure 5 , in the case $n=1$ (with $\alpha=.3$ ).

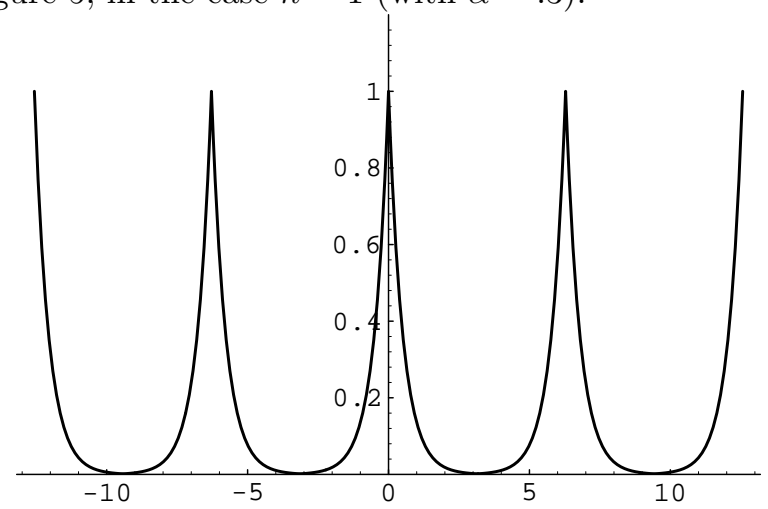

Figure 5: Approximate low-pass filter for "exponential-type" wavelet with 1 vanishing moment

The case $n=1$ is not very interesting numerically because $\hat{\varphi}$ is nondifferentiable at $\xi=0$ and the $m_{0}$ filter has slow decay. We shall then show the case $n=5, \alpha=.3$ for which the low-pass filter is shown in figure 6 .

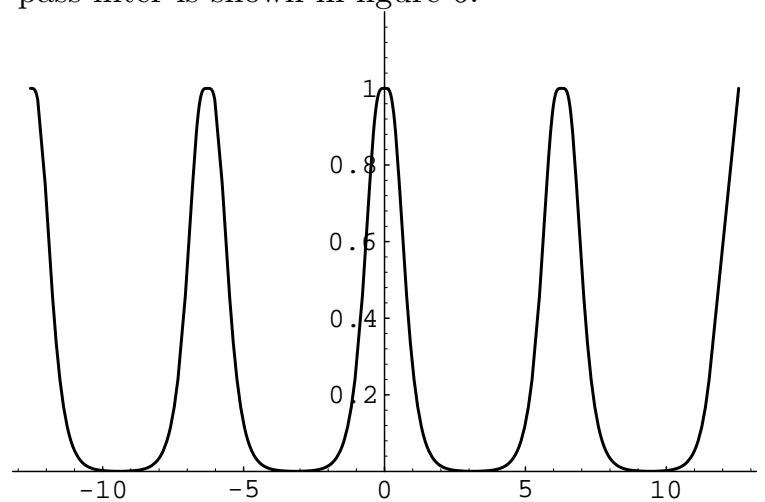

Figure 6: Approximate low-pass filter for "exponential-type" wavelet with 5 vanishing moments and the 16 top low-pass and high-pass filter coefficients are given by:

$h_{k}$ coefficients:

$\{0.2608909,0.21193501,0.11802165,0.047464751$,

$0.012065044,-0.001261182,-0.004691885,-0.004500855$,

(89)

$-0.0034217616,-0.0023426834,-0.0015318216,-0.00096772754$,

$-0.00060530854,-0.00037287531,-0.00023103465,-0.00014240093\}$

$g_{k}$ coefficients:

$$
\begin{aligned}
& \{0.7391091,-0.21193501,-0.11802165,-0.047464751, \\
& -0.012065044,0.001261182,0.004691885,0.004500855, \\
& 0.0034217616,0.0023426834,0.0015318216,0.00096772754, \\
& 0.00060530854,0.00037287531,0.00023103465,0.00014240093\}
\end{aligned}
$$

It is worth noticing that all such coefficients are easy to obtain numerically.

4.3. The Cauchy wavelets. The same filters as before can be used to work with the wavelets that were used by T. Paul in a quantum mechanical context (they are canonically associated with the radial Schrodinger equation for the Hydrogen atom 
for instance). They are of the form

$$
\hat{\psi}_{n}(\xi)= \begin{cases}\frac{1}{(n-1) !} \xi^{n} e^{-\xi} & \text { for positive values of } \xi \\ 0 & \text { otherwise }\end{cases}
$$

$n=1, \ldots \infty$ then controls the number of vanishing moments, and $\hat{\psi}_{n}(\xi)$ has exponential decay for all $n$. A direct computation yields the corresponding scaling function

$$
\hat{\varphi}_{n}(\xi)= \begin{cases}e^{-\xi} \sum_{p=0}^{n-1} \frac{1}{p !} \xi^{p} & \text { for positive values of } \xi \\ 0 & \text { otherwise }\end{cases}
$$

The first one is particularly interesting since it is canonically related with the Cauchy kernel. Indeed, the scaling function coefficients of a function $f(x) \in H^{2}(\mathbb{R})$ form an analytic function of $z=b+i a$, that is the analytic continuation $f(z)$ of $f(x)$ to the upper half-plane. The corresponding wavelet transform is then (up to a factor $a$ ) the derivative of $f(z)$ with respect to its imaginary part: $T_{f}(b, a)=-a \partial_{a} f(b+i a)$.

The previous filters can then be used to get a fast approximate algorithm for wavelet transform with such wavelets. Of course, adapted filters can also be obtained by using directly the formula yielding the $\tilde{m}_{i}$ filters. However, $\hat{\phi}_{n}$ being discontinuous at the origin for any $n$, such filters are not suitable for numerical use since they have slow decay.

5. Conclusions. We have described in this paper a method that associates a pair of ( $2 \pi$-periodic) filters with a Littlewood-Paley (or dyadic wavelet) decomposition, yielding a pyramidal algorithm for the computation of a corresponding approximate transform.

We have in particular shown that in the case where the Littlewood-Paley decomposition comes from a "linear scheme" of infinitesimal wavelet analysis (as described in [2]), such filter fulfill a kind of "linear" QMF relation, leading to simple reconstruction formulas from the approximate coefficients. Our main result was an estimate of the accuracy of the approximate algorithm. The problem of finding approximate filters was transformed into a minimization problem having a unique solution. Of course when there already exist a pair of $2 \pi$-periodic filters naturally associated with the wavelet, this solution coincides with it.

In the case of the "linear scheme" of infinitesimal wavelet analysis, we also got explicit expressions for approximate filters. It is to be noticed that in some cases, the error estimates go to zero as the scale becomes larger and larger. This is due to the fact that the wavelet transform is sampled at a fixed sampling frequency, independently of the scale. In such cases, the redundancy of the wavelet transform implies the stability of the algorithm.

As in the case of usual multiresolution analysis, the localization (i.e. decay properties) of the approximate filters is directly related to the regularity of the scaling function.

We also have discussed some simple examples, in particular those of the LOG and DOG wavelets in the linear scheme, familiar to computer vision specialists, and wavelets of exponential type. If the corresponding scaling functions are sufficiently well localized in the Fourier space, good error estimates are obtained.

Let us notice that $n$-dimensional generalizations of our method with the tensorproduct construction of filters are straightforward. 
Acknowledgements. This research was partly supported by a CNRS/CONICET cooperation agreement, and the Physics department of Université de Provence, Marseille. We also wish to thank A. Grossmann and Ph. Tchamitchian, for stimulating discussions.

\section{REFERENCES}

[1], Ten lectures on wavelets, CBMS-NSF series, SIAM (1992).

[2] , Continuous wavelet decompositions, multiresolution and contrast analysis, to appear at SIAM J. Math. An. (1993).

[3] , Littlewood-Paley theory and the study of function spaces, CBMS-NSF Regional conference series (1992).

[4] , A real-time algorithm for signal analysis with the help of the wavelet transform, in "Wavelets, time-frequency methods and phase space", J.M. Combes \& al Eds, IPTI, Springer (1989), 286-297.

[5] , Multiresolution representations using the autocorrelation functions of compactly supported wavelets, Proceedings of the international conference "Wavelets and applications", Toulouse, Y. Meyer and S. Roques Eds, Editions Frontières (1993).

[6] , Ondelettes et opérateurs: I Ondelettes, (1989), Hermann.

[7] , Affine wavelets: wedding the Atrous and the Mallat algorithms, IEEE Trans. Acoust. Speech. Processing, to appear. 